\title{
Sorted Chase Detection Algorithm for MIMO System
}

\author{
Liu Li ${ }^{1, a}$, Wang Jinkuan ${ }^{1, b}$, Liu Fulai ${ }^{2, ~ c}$, Song $\mathrm{Xin}^{2, \mathrm{c}}$, Han Yinghua ${ }^{2, \mathrm{c}}$, \\ Wang Yuhuan ${ }^{1, c}$ \\ ${ }^{1}$ School of Information Science \& Engineering, Northeastern University, Liaoning, China \\ ${ }^{2}$ Engineering Optimization and Smart Antenna Institute, Northeastern University at Qinhuangdao, \\ Hebei, China \\ aliuliqhd@126.com, bwjkqhd@126.com, ㄷneuqdl@163.com
}

Keywords: MIMO detection, sorted QR decomposition, error propagation, Chase detection

\begin{abstract}
In multiple-input multiple-output wireless communication system, the order of symbol detection in Chase framework is critical because of error propagation. In order to improve chase detector's performance, sorted chase detection algortihm to reduce error propagation is proposed here. By setting parameters at different values, trade-off between performance and complexity can be obtained properly. Simulation experiment results show the validity of proposed algorithm.
\end{abstract}

\section{Introduction}

In multiple-input multiple-output (MIMO) wireless system, communication capacity can be increased enormously without increasing the bandwidth or transmitted power [1]. Serial data streams are converted to parallel and transmitted on different antenna in MIMO system. In order to decode symbols, efficient signal detection algorithms for MIMO systems have attracted much interest in recent years [2,3], and many detection techniques had been proposed such as line detection, maximum likelihood (ML) detection, Bell-Labs layered space-time (VBLAST) detection [4], etc.

ML detection algorithm is the optimum detection algorithm at the performance of bit-error rate (BER), but the computational complexity growing exponentially with the order of modulation and the number of transmit-antennas, which makes the ML detection algorithm difficulty to use in practice. Other detections have low computation complexity comparing with ML detection; meanwhile the performance is also lower than ML detection. A lot of efforts have been put into the search of detection algorithms achieving ML or near-ML performance with lower complexity, such as tree search algorithm, lattice reduction, chase detector, and so on.

Chase detector for MIMO detection is combined with a list detector and parallel banks of subdetectors [5]. Chase detector could be regarded as the unified framework of existing detectors including ML, successive interference cancellation detection (SIC), and VBLAST detection. From the detection mechanism of chase detector, it can be regarded as SIC detection algorithm in essence. When wrong symbol is selected in the first detection stage, it will lead to error propagation in later sub-detectors.

In order to reduce error propagation and calculating complexity, a sorted chase detection algorithm is proposed here which use QR decomposition to create candidate list without calculation of pseudo-inverses of channel matrix and an adjustable parameters to restrain error producing further more. These schemes can reduce the error in initially detection step by selecting the symbols with minimum error into the list. Trade-off between performance and complexity can be obtained by proposed detection algorithm. 


\section{Proposed Detection Algorithm}

System Model. Un-coded MIMO system is consisted of $N$ transmit antennas at the transmitter and $M$ antennas at the receiver ( $M \geq N$ ). The wireless channel is assumed to be quasi-static, so the received signal vector can be represented as

$$
\boldsymbol{y}(t)=\boldsymbol{H}(t) \boldsymbol{x}(t)+\boldsymbol{n}(t)
$$

where $\boldsymbol{x}(t)=\left[x_{1}(t), \ldots, x_{N}(t)\right]^{\mathrm{H}}, \boldsymbol{y}(t)=\left[y_{1}(t), \ldots, y_{M}(t)\right]^{\mathrm{H}}$ and $\boldsymbol{n}(t)=\left[n_{1}(t), \ldots, n_{M}(t)\right]^{\mathrm{H}}$ is the sent symbol, the received symbol and the noise symbol, respectively. The symbols $x_{n}(t)$ and the noise $n_{m}(t)$ are mutually uncorrelated, zero-mean random processes, which variances is $\mathrm{E}\left\{\left|x_{n}(t)\right|^{2}\right\}=1$ and $\mathrm{E}\left\{\left|n_{m}(t)\right|^{2}\right\}=\sigma^{2}$, respectively. The element $h_{i j}$ of $\boldsymbol{H}(t)$ represents channel gains between the $j^{\text {th }}$ transmitter and the $i^{\text {th }}$ receiver antennas at the discrete time $\mathrm{t}$.

Original Chase Detection Framework. The Chase detector defines a framework for existing MIMO detection algorithms. Five steps are contained in Chase detector, as shown in Fig.1.

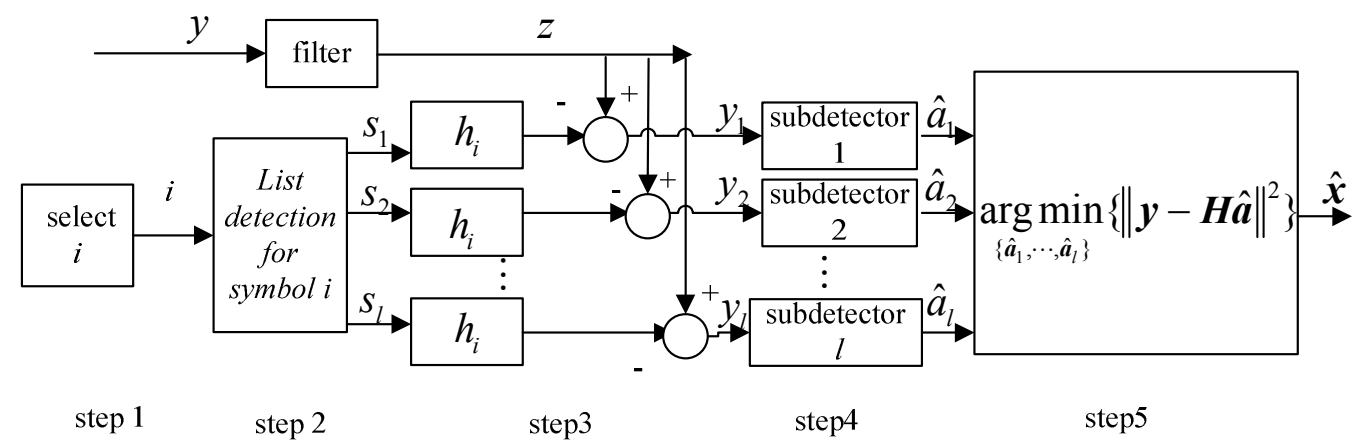

Fig. 1. Block diagram of the Chase detector

Five steps are outlined in the framework:

Step1. Identify which symbol to be detected first according to the channel matrix $\boldsymbol{H}$.

Step2. Generate a sorted list $L=\left\{s_{1}, \cdots s_{l}\right\}$ of candidate values for $i^{\text {th }}$ symbol, defined as the $l$ elements of the alphabet nearest to $z_{i}$, where $\boldsymbol{z}=\boldsymbol{G} \boldsymbol{y}$ is the output of linear filter.

Step3. Generate a set of $l$ residual vectors $\left\{y_{1}, \cdots y_{l}\right\}$ by canceling the contribution to $\boldsymbol{y}$ from the $i^{\text {th }}$ symbol, assuming each candidate from the list is, in turn, correct: $\boldsymbol{y}_{j}=\boldsymbol{y}-\boldsymbol{h}_{i} \boldsymbol{s}_{j}$

Step4. Assign each of $\left\{y_{1}, \cdots y_{l}\right\}$ to its own independent sub-detector to detect the remaining $N_{t}-1$ symbols (all but the $i^{\text {th }}$ symbol).

Step5. Choose the candidate $\hat{\boldsymbol{a}}$ with the minimum mean square error as the final hard decision

$$
\hat{\boldsymbol{x}}=\underset{\left\{\hat{\boldsymbol{a}}_{1}, \cdots, \hat{\boldsymbol{a}}_{l}\right\}}{\arg \min }\left\{\|\boldsymbol{y}-\boldsymbol{H} \hat{\boldsymbol{a}}\|^{2}\right\}
$$

Improved Chase Detection Algorithm. In Chase detector, which symbol to be detected first is critical to all the overall performance. Maximum post SNR ordering methods is proved to be the optimal detection order in V-BLAST with iterative pseudo-inverses calculating. For reducing error propagation, symbols should be detected with the optimal order. In order to select the first detected symbol correctly with lower complexity, an improved Chase detection algorithm is proposed here, the diagram is shown in fig.2. The proposed detector is different to the original Chase detector in step1 and step2. 
In order to reduce the error produced in the first detection step, a sorted QR decomposition is performed firstly, and a new parameter $q$ is set in step 1 for deciding the numbers of the first detected layers. Instead of selecting only one layer, $q$ layers are selected and ML detection is performed for the selected layers. The SNR of $i^{\text {th }}$ layer can be described

$$
\mathrm{SNR}_{i}=\frac{E\left[\left|x_{i}\right|^{2}\right]}{E\left\{\bar{n}_{i}\right\}^{2}\left[\boldsymbol{R}^{-1}\left(\boldsymbol{R}^{-1}\right)^{H}\right]_{i, i}}=\frac{1}{\sigma^{2}\left\|(\boldsymbol{R})^{-1}{ }_{i:}\right\|_{2}} \sim\left\|(\boldsymbol{R})_{i:}\right\|_{2}
$$

where, [. $]_{i, i}$ is the $i^{\text {th }}$ diagonal component of matrix [.], $\left\|(R)^{-1}{ }_{i:}\right\|_{2}$ is the 2-norm of the $i^{\text {th }}$ row of $\boldsymbol{R}^{-1}$.

So the optimized ordered rule of maximum $S N R$ is equivalent to the order of row 2-norm of $\boldsymbol{R}$. The detection order can be obtained by QR decomposition of channel $\boldsymbol{H}$, sorted and QR decomposition of $\boldsymbol{R}$ without calculating pseudo-inverses of the channel matrix $\boldsymbol{H}$. Here GramSchmidt method is used to sorted QR decomposition.

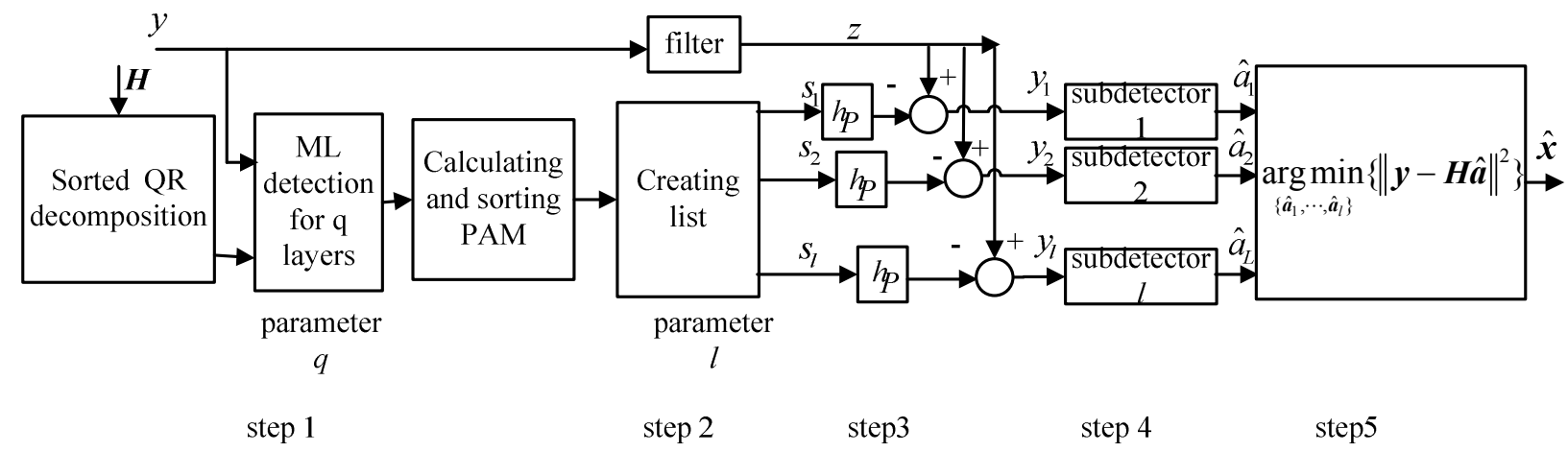

Fig. 2. Block diagram of modified Chase detectors

After the $q$ layers (their layer's numbers are $N, N-1, \cdots, N-q+1$, respectively) are performed ML search, the partial accumulated metrics (PAM) are calculated and sorted as

$$
\operatorname{PAM}(\lambda)=\sum_{i=N-q+1}^{N}\left(\bar{y}_{i}-\sum_{j=i}^{N} \overline{\boldsymbol{R}}_{i, j} \hat{x}_{j}\right)^{2}
$$

where $\lambda=1, \cdots,|\Omega|^{q}$. $\Omega$ is the set of modulation constellation, $|\Omega|$ is the cardinality of modulation constellation. Without loss of generality that the symbol with lower index has smaller metrics, $\operatorname{PAM}(1) \leq \operatorname{PAM}(2) \leq \cdots \leq \operatorname{PAM}(\lambda)$. Now we get $|\Omega|^{q}$ available partial candidate paths. Because of the property of the exhaustive search algorithm, we can detect symbols at this stage and guarantee the performance without error propagation. Then these sequences are sorted with the partial accumulated metrics.

To reduce error propagation further, in step2, another parameter $l$ is set for defining candidate list length. The list of partial candidates is generated in which $l$ candidate signal sequences with smaller partial accumulated metrics are selected in step2. More candidates are selected to look for more possible solution.

In step3 and step4 the searching for left layers are performed with ZF criteria sorted QR decomposition algorithm. .

In the last step, the candidate signal sequence with the smallest accumulated metrics is quantization, decision and re-arranged as the transmitted signal.

$$
\hat{\boldsymbol{x}}=\underset{\boldsymbol{x}}{\arg \min }\left(\|\tilde{\boldsymbol{y}}-\boldsymbol{R} \hat{\boldsymbol{x}}\|^{2}\right)=\underset{\boldsymbol{x}}{\arg \min }\left(\sum_{i=1}^{N}\left(\tilde{y}_{i}-\sum_{j=i}^{N} \boldsymbol{R}_{i, j} \hat{x}_{j}\right)^{2}\right)
$$


The maximum likelihood detection of the transmitted signal can be formulated as finding

$$
\hat{\boldsymbol{x}}=\underset{x \in\{\Omega\}^{N}}{\arg \min }\left\{\|\boldsymbol{y}-\boldsymbol{H} \boldsymbol{x}\|^{2}\right\}
$$

\section{Simulation Experiments}

MIMO system with $N=4, M=4$ and QPSK modulation is considered here. Channel is assumed to be Rayleigh flat-faded and no correlation between sub-channels. Detection performance of proposed Chase detector compares with VBLAST and ML detection (MLD). The BER curves are shown in Figure 3.

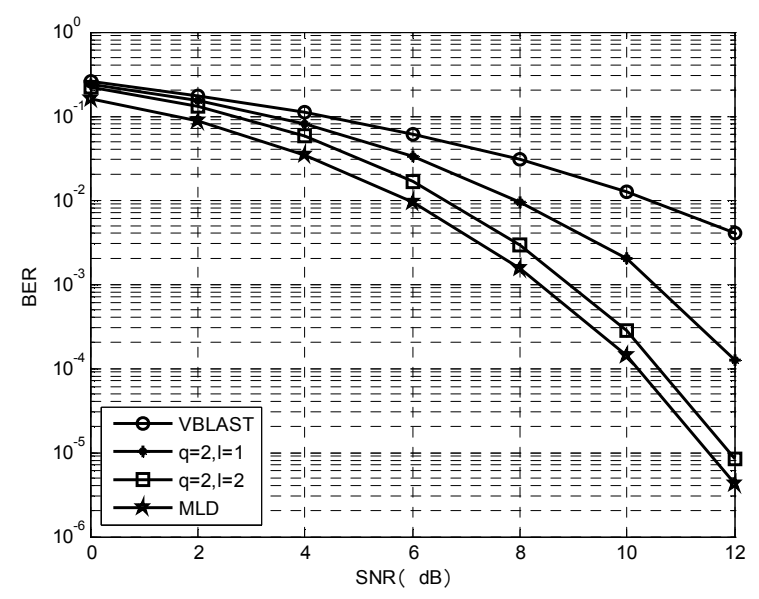

Fig. 3. BER comparison of detection performance

From the comparison results, it can be found that the BER performance of the proposed algorithm can be improved as $q$ and $l$ increases. With increasing of the two parameters, detection performance gains improving with calculating complexity much lower than ML detection.

\section{Summary}

For reducing the error propagation at the first detection layers in chase detection, improved sorted QR algorithm has been used to get the detection order without calculating pseudo-inverses of the channel matrix. The proposed chase detection had set two adjustable parameters to reduce error propagation. Performance has been improved by modifying the numbers of maximum likelihood detection layers and the list length. Simulation results show that the presented algorithm is superior to VBLAST algorithm.

\section{Acknowledgments}

This work is supported by the National Natural Science Foundation of China under Grant No. 60904035, 61004052 and 61104005, the Natural Science Foundation of Heibei Province of China grant No. F2011501052. 


\section{References}

[1] E. Telatar, Capacity of multi-antenna Gaussian channel. Europe. Trans. Tele., 10(11) (1999) 585-595.

[2] C. E. Chen, W. H. Sheen, A new Lattice Reduction Algorithm for LR-Aided MIMO Linear Detection, IEEE Trans. on Wirel. Commun. 10 (2011) 2417.

[3] R. Y. Chang, W. H. Chung, Best-First Tree Search with Probabilistic Node Ordering for MIMO Detection: Generalization and Performance-Complexity Tradeoff, IEEE Trans. Wirel. Comm. 11 (2012) 780-789

[4] P. W. Wolniansky, G. J. Foschini, G. D. Golden, R. A. Valenzuela, V-BLAST: An Architecture for Realizing Very High Data Rates Over the Rich-Scattering Wireless Channel, ISSSE. 295300. (1998).

[5] W. Waters, and J.R. Barry, The Chase Family of Detection Algorithm for Multiple-Input Multiple-Output Channel. IEEE Trans. on Signal Processing, 56 (2008) 739-747 